\title{
Towards Thermodynamics with Generalized Uncertainty Principle
}

\author{
Ahmed Farag Ali ${ }^{1,2}$ and Mohamed Moussa ${ }^{2}$ \\ ${ }^{1}$ Center for Fundamental Physics, Zewail City of Science and Technology, Giza 12588, Egypt \\ ${ }^{2}$ Department of Physics, Faculty of Sciences, Benha University, Benha 13518, Egypt
}

Correspondence should be addressed to Ahmed Farag Ali; ahmed.ali@fsc.bu.edu.eg

Received 9 December 2013; Revised 12 January 2014; Accepted 13 January 2014; Published 25 February 2014

Academic Editor: Elias C. Vagenas

Copyright (c) 2014 A. Farag Ali and M. Moussa. This is an open access article distributed under the Creative Commons Attribution License, which permits unrestricted use, distribution, and reproduction in any medium, provided the original work is properly cited. The publication of this article was funded by SCOAP ${ }^{3}$.

\begin{abstract}
Various frameworks of quantum gravity predict a modification in the Heisenberg uncertainty principle to a so-called generalized uncertainty principle (GUP). Introducing quantum gravity effect makes a considerable change in the density of states inside the volume of the phase space which changes the statistical and thermodynamical properties of any physical system. In this paper we investigate the modification in thermodynamic properties of ideal gases and photon gas. The partition function is calculated and using it we calculated a considerable growth in the thermodynamical functions for these considered systems. The growth may happen due to an additional repulsive force between constitutes of gases which may be due to the existence of GUP, hence predicting a considerable increase in the entropy of the system. Besides, by applying GUP on an ideal gas in a trapped potential, it is found that GUP assumes a minimum measurable value of thermal wavelength of particles which agrees with discrete nature of the space that has been derived in previous studies from the GUP.
\end{abstract}

\section{Introduction}

One of the intriguing predictions of various frameworks of quantum gravity such as string theory and black hole physics is the existence of a minimum measurable length. This has given a rise to the so-called generalized uncertainty principle (GUP) or, equivalently, modified commutation relations between position coordinates and momenta [1-8]. This can be understood in the context of string theory since strings cannot interact at distances smaller than their size [9]. The GUP is represented by the following form [10-12]:

$$
\begin{array}{r}
\Delta x_{i} \Delta p_{i} \geq \frac{\hbar}{2}\left[1+\beta\left((\Delta p)^{2}+\langle p\rangle^{2}\right)\right. \\
\left.+2 \beta\left(\Delta p_{i}^{2}+\langle p\rangle^{2}\right)\right],
\end{array}
$$

where $p^{2}=\sum_{j} p_{j} p_{j}, \beta=\beta_{0} /\left(M_{p} c\right)^{2}=\beta_{0}\left(\ell_{p}^{2} / \hbar^{2}\right), M_{p}=$ Planck mass, and $M_{p} c^{2}=$ Planck energy. Inequality (1) is equivalent to the following modified Heisenberg algebra [10]:

$$
\left[x_{i}, p_{j}\right]=i \hbar\left(\delta_{i j}+\beta \delta_{i j} p^{2}+2 \beta p_{i} p_{j}\right) \text {. }
$$

This form ensures, via the Jacobi identity, that $\left[x_{i}, x_{j}\right]=0=$ $\left[p_{i}, p_{j}\right][11]$. Recently, a new form of GUP was proposed in [13, 14], which predicts a maximum observable momenta beside the existence of a minimal measurable length. This new form was constructed to be consistent with doubly special relativity theories (DSR) [15], string theory, and black holes physics [17]. It ensures $\left[x_{i}, x_{j}\right]=0=\left[p_{i}, p_{j}\right]$, via the Jacobi identity. This new form of the GUP is given as follows:

$$
\begin{array}{r}
{\left[x_{i}, p_{j}\right]=i \hbar\left[\delta_{i j}-\alpha\left(p \delta_{i j}+\frac{p_{i} p_{j}}{p}\right)\right.} \\
\left.+\alpha^{2}\left(p^{2} \delta_{i j}+3 p_{i} p_{j}\right)\right],
\end{array}
$$

where $\alpha=\alpha_{0} / M_{p} c=\alpha_{0} \ell_{p} / \hbar, M_{p}=$ Planck mass, $\ell_{p}=$ Planck length, and $M_{p} c^{2}=$ Planck energy. The most important consequence of this model is the discreteness of space which confirms that all measurable lengths are quantized in units of a fundamental minimum measurable 
length $[13,14]$. As a result, according to (3), GUP modifies the physical momentum $[13,14,16]$ :

$$
x_{i}=x_{0 i}, \quad p_{i}=p_{0 i}\left(1-\alpha p_{0}+2 \alpha^{2} p_{0}^{2}\right) .
$$

Then it is natural to expect that this would result in considerable modifications in the dispersion relation to be in the form $[17,18]$

$$
E^{2}(p)=p^{2} c^{2}(1-\alpha p)^{2}+m^{2} c^{4} .
$$

This definitely would affect a host of quantum phenomena. In a series of earlier papers, various applications of the new model of GUP were investigated on atomic physics, condensed matter physics, preheating phase of the universe, and black holes at LHC [16-29]. Also, one of the authors investigated its effect with the Liouville theorem in statistical mechanics [25]. So according to Liouville theorem, it should be stated that the number of states inside volume of phase space does not change with time evolution in presence of the GUP. So GUP should modify the density states and this implies essential effects on the statistical and thermodynamic properties of any physical system. According to [25], the modification in the number of quantum states per momentum space volume should be as follows:

$$
\frac{V}{(2 \pi \hbar)^{3}} \int d^{3} p \rightarrow \frac{V}{(2 \pi \hbar)^{3}} \int \frac{d^{3} p}{(1-\alpha p)^{4}} .
$$

For more details about proving the above equation, (6), we suggest consulting [25]. The upper bounds on the GUP parameter $\alpha$ have been derived in [16]. It was suggested that these bounds can be measured using quantum optics techniques and gravitational wave techniques in [30,31]. Recently, Bekenstein [32,33] proposed that quantum gravitational effects could be tested experimentally; he suggests "a tabletop experiment which, given the state-of-the-art ultrahigh vacuum and cryogenic technology, could already be sensitive enough to detect Planck scale signals" [32]. This would put several quantum gravity predictions to test in the laboratory [30,31]. Definitely, this is considered as a milestone in the road of quantum gravity phenomenology.

It appears that the total number of microstates is increased due to GUP correction. It is worth mentioning that there are other theories that predict other forms of modified dispersion relations. These models were introduced in [3437] and suggest a violation in Lorentz symmetry at some energy scale which could be quantum gravity scale. Their thermodynamical implications have been studied in $[34,35]$. Besides there are other approaches that suggest the existence of an independent observer scale which could be a Planck energy scale and this has been formulated to the so-called doubly special relativity (DSR) [38-41]. We can see that DSR is a generalization of special relativity. DSR transformation has several approaches; one of the important approaches is considered in $[42,43]$. The interesting thing about DSR is that it does not violate Lorentz symmetry and the basic postulates of special relativity are still satisfied, but it introduces an upper limit of energy. In this approach, the dispersion relation for massless particles does not change, modifications take place only for massive particles, and according to [44, 45] there is no change in the density states. However, there remains a considerable change in the partition function due to the presence of an upper bound in energy of particles.

In this paper we continue investigating the modification of thermodynamics but this time in the presence of GUP, proposed by Ali et al. $[13,14,16]$. We consider two cases: ideal gas and photon gas. It is worth mentioning that a different version of GUP has been studied with ideal gas in $[46,47]$. The derivation of the expression for partition function in the presence of GUP is the most crucial result of our paper. We calculated analytic expressions for the pressure, internal energy, entropy, and specific heat of the considered systems. We show the difference between the GUP case and standard case. Our results go back to the standard forms derived when we set $\alpha=0$.

\section{Ideal Gas}

Consider $N$ noninteracting particles, obeying MaxwellBoltzmann statistics and are confined in a volume $V$ at temperature $T$. For a canonical ensemble, the thermodynamics of this system can be derived from the total partition function $[48,49]$ :

$$
\mathscr{Z}(N)=\frac{1}{N !}[\mathscr{Z}(1)]^{N} .
$$

We have considered classical Maxwell-Boltzmann statistics along with Gibb's factor and $Z(1)$ is the single partition function given by

$$
\mathscr{Z}(1)=\frac{1}{(2 \pi \hbar)^{3}} \int d^{3} r \int e^{-\beta \epsilon} d^{3} p,
$$

where $\beta=1 / K T$ and $\epsilon$ is the total energy. The integral over the coordinate yields the volume of the gas. Equation (8) can be rewritten in the form

$$
\mathscr{Z}(1)=\frac{V}{(2 \pi \hbar)^{3}} \int e^{-\beta\left(E-m c^{2}\right)} d^{3} p,
$$

where $E$ and $m$ are the total relativistic energy and the rest mass of gaseous particles. To calculate the quantum gravitational effect, the GUP can be considered in the phase space analysis by two equivalent pictures: (a) considering deformed commutation relations (i.e., deformed the measure of integration) simultaneously with the nondeformed Hamiltonian function or (b) calculating canonical variables on the GUP-corrected phase space which satisfy the standard commutative algebra (i.e., nondeformed standard measure of integration), but the Hamiltonian function now gets deformed. These two pictures are related to each other by the Darboux theorem in which it is quite possible to find canonical coordinates on the symplectic manifold which satisfy standard Heisenberg algebra (we thank the referee for paying our attention for this important note). In this paper we are going to consider deformed measure of integration 
with nondeformed Hamiltonian function. According to (6), we find that (9) takes the form

$$
\mathscr{Z}(1)=\frac{V}{2 \pi^{2} \hbar^{3}} e^{\beta m c^{2}} \int_{0}^{\infty} e^{-\beta\left[p^{2} c^{2}+m^{2} c^{4}\right]^{1 / 2}} \frac{p^{2} d p}{(1-\alpha p)^{4}} .
$$

In order to solve this integral, use the substitution

$$
p=m c \sinh x \text {. }
$$

In first order approximation in $\alpha$, we can prove that

$$
\frac{p^{2} d p}{(1-\alpha p)^{4}} \simeq m^{3} c^{3} \cosh x \sinh ^{2} x(1+4 \alpha m c \sinh x) d x
$$

As one might expect, the approximation used so far, that is, $1 / \alpha>p$, breaks down around a maximum measurable energy $(\sim 1 / \alpha)$ and we need an exact treatment to describe the solution around this scale. Therefore, one can only trust a perturbative solution where $p \alpha \ll 1$ (we thank the referee for pointing out this important note). Then the expression which remains to be calculated is

$$
\begin{aligned}
\mathscr{Z}(1)= & \frac{V}{2 \pi^{2} \hbar^{3}} m^{3} c^{3} e^{\beta m c^{2}} \\
& \times \int_{0}^{\infty} e^{-\beta m c^{2} \cosh x} \cosh x \sinh ^{2} x \\
& \times(1+4 \alpha m c \sinh x) d x .
\end{aligned}
$$

This integration can be calculated to take the form

$$
\begin{aligned}
\mathscr{Z}(1)= & \frac{V}{2 \pi^{2} \hbar^{3}} m^{3} c^{3} \frac{e^{u}}{u} K_{2}(u) \\
& +4 \alpha \frac{V}{\pi^{2} \hbar^{3}} \frac{m^{4} c^{4}}{u^{2}}\left(1+\frac{3}{u}+\frac{3}{u^{2}}\right),
\end{aligned}
$$

where $u=\beta m c^{2}$ and $K_{n}(u)$ is the second order modified Bessel function [50]. Setting $\alpha=0$ the partition function reduces to the usual case [49]. In nonrelativistic limit, $u \rightarrow$ $\infty$, or the thermal energy of the particle is very small compared to the rest mass; we have $k_{2}(u) \approx \sqrt{\pi /(2 u)} e^{-u}$; thus

$$
\mathscr{Z}(1)=\frac{V}{\hbar^{3}}\left(\frac{m K T}{2 \pi}\right)^{3 / 2}\left[1+16 \alpha\left(\frac{m K T}{2 \pi}\right)^{1 / 2}\right] .
$$

Analogously, for very high temperature $u \rightarrow 0$, which means ultra-relativistic particles in which the thermal energy is much greater than the rest mass. The modified Bessel function behaves like $k_{2}(u) \approx 2 / u^{2}$ and (14) takes the form

$$
\mathscr{Z}(1)=\frac{V}{\pi^{2}}\left(\frac{K T}{\hbar c}\right)^{3}\left[1+12 \alpha \hbar\left(\frac{K T}{\hbar c}\right)\right] .
$$

It should be noted that as $\alpha \rightarrow 0$, we get back the results of special theory of relativity thermodynamics [49]. The partition function relates the microscopic properties with the thermodynamic (macroscopic) behavior of the physical systems. Using the expression of the partition function, we will go on to study various thermodynamic quantities of ideal gases with GUP effects. The free energy of the system is defined as

$$
F=-\frac{1}{\beta} \ln \mathscr{Z}(N) \text {. }
$$

Using Stirling's formula $\ln N ! \approx N \ln N-N$, the free energy for the ideal gas is given by

$$
F=-N \frac{m c^{2}}{u}\left[1+\ln \left(\frac{V}{2 \pi^{2} \hbar^{3}} \frac{m^{3} c^{3}}{N}\right)+\ln F_{1}\right],
$$

where

$$
F_{1}=\frac{e^{u}}{u} k_{2}(u)+8 \alpha \frac{m c}{u^{2}}\left(1+\frac{3}{u}+\frac{3}{u^{2}}\right) .
$$

Using the expression of the free energy (18), the pressure can be obtained by the relation

$$
P=-\left.\frac{\partial F}{\partial V}\right|_{N, T}=\frac{N K T}{V} .
$$

We have the same result as in the relativistic case which means that the pressure does not change with considering the GUP. The chemical potential is given by the relation

$$
\mu=\left.\frac{\partial F}{\partial N}\right|_{T, V}=-K T\left[\ln \left(\frac{V}{2 \pi^{2} N} \frac{m^{3} c^{3}}{\hbar^{3}}\right)+\ln F_{1}\right] .
$$

We can evaluate the entropy using free energy by the relation

$$
\begin{aligned}
S & =-\left.\frac{\partial F}{\partial T}\right|_{N, V}=\left.\frac{K u^{2}}{m c^{2}} \frac{\partial F}{\partial u}\right|_{N, V} \\
& =N K\left[1+\ln \left(\frac{V}{2 \pi^{2} \hbar^{3}} \frac{m^{3} c^{3}}{N}\right)+\ln F_{1}\right]+N K \frac{F_{2}}{F_{1}},
\end{aligned}
$$

where

$$
\begin{aligned}
F_{2}= & {\left[k_{1}(u)+\left(\frac{3}{u}-1\right) k_{2}(u)\right] e^{u} } \\
& +8 \alpha \frac{m c}{u^{2}}\left[2+\frac{9}{u}+\frac{12}{u^{2}}\right] .
\end{aligned}
$$

By setting $\alpha=0$, we get back the usual results. We see that the new terms, due to quantum gravity, carry a positive sign. This indicates that the modified entropy grows faster than standard case by increasing temperature. The elevation in the number of accessible microstates in high momentum regime, or in Planck scale, leads to increase in corresponding microcanonical entropy of the system. This may be interpreted that the existence of the GUP implied an additional repulsive force between the constitutes of the gas which leads to an increase in the entropy of the system. Modification in the expression of internal energy is expected due to change in the free energy. It can be evaluated from the following relation:

$$
U=F+T S=F+\frac{m c^{2}}{K u} S=N K T \frac{F_{2}}{F_{1}} .
$$




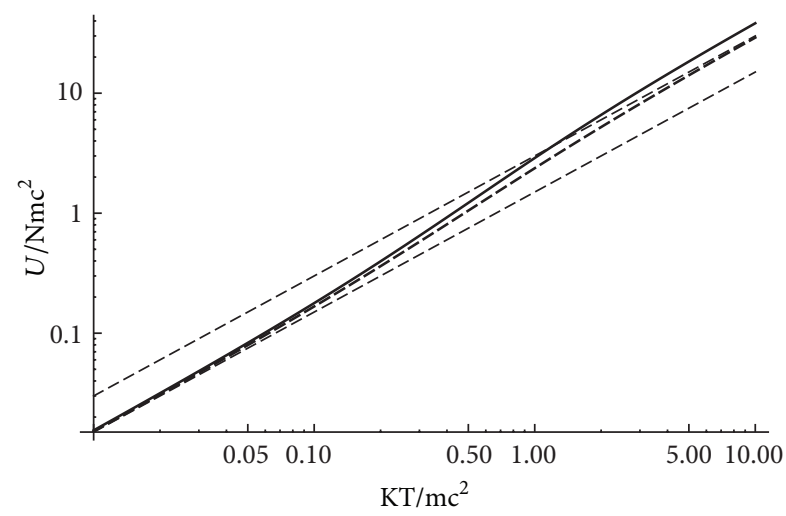

FIGURE 1: The dashed curves represent the unmodified internal energy and its non- and ultrarelativistic limits. The solid curve represents the modified internal energy.

If we put $\alpha=0$, we obtain the standard special relativity internal energy expression. Without quantum gravity effect, $u \ll 1$ yields ultrarelativistic limit $U=3 N K T$ and $u \rightarrow \infty$ yields $U=(3 / 2) N K T$. Figure 1 shows relativistic internal energy compared with a modified one (solid curve). It is observable from this figure that the GUP-modified internal energy grows faster than the relativistic case. At high temperature, or in ultrarelativistic regime, the modified internal energy becomes greater than the asymptotic value. The increase in entropy, due to quantum gravity, leads to an increase in the internal energy as it would be expected. Each of the entropy and the internal energy are not measurable quantities directly. We can detect the effect of GUP through other thermodynamic quantities. The specific Heat $C_{V}$ can be detected experimentally. It is defined by

$$
\begin{aligned}
C_{V} & =\left(\frac{\partial U}{\partial T}\right)_{V}=-\frac{K u^{2}}{m c^{2}}\left(\frac{\partial U}{\partial u}\right)_{V} \\
& =N K \frac{u}{F_{1}}\left[\frac{F_{2}}{u}\left(1-\frac{F_{2}}{F_{1}}\right)-F_{3}\right],
\end{aligned}
$$

where

$$
\begin{aligned}
F_{3}= & {\left[2 k_{1}(u)\left(1-\frac{1}{u}\right)-k_{2}(u)\left(2-\frac{5}{u}+\frac{9}{u^{2}}\right)\right] } \\
& \times e^{u}-8 \alpha \frac{m c}{u^{3}}\left[4+\frac{27}{u}+\frac{48}{u^{2}}\right] .
\end{aligned}
$$

It is straightforward to see from (25) that we get back the usual specific heat for $\alpha=0$. In Figure 2 the dashed curves represent the usual relativistic specific heat; it grows from the nonrelativistic limit value $C_{V}=(3 / 2) N K T$ up to an ultrarelativistic limit value $C_{V}=3 N K T$. The solid curve represents the modified specific heat. It is clear that the new specific heat grows faster than the relativistic one with increasing temperature. We can see from Figure 2 that in ultrarelativistic range the modified specific heat is greater than the corresponding relativistic one and it approaches a constant value. This approach is specified for ideal gas particles that move in free regime in a relativity small

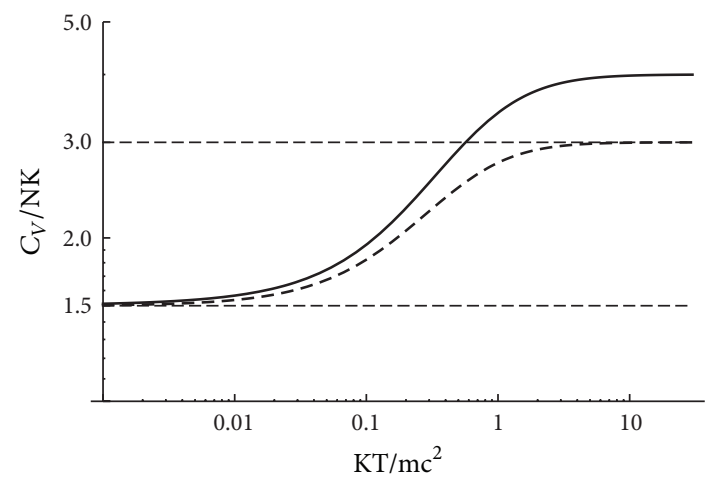

FIGURE 2: The dashed curves represent the unmodified specific heat and its non- and ultrarelativistic limits. The solid curve represents the modified specific heat.

pressure. But if the gas particle motion is restricted in a small volume, then the particles may be considered in a potential trap. Now we will move to study the effect of quantum gravity on a ideal gas in potential trap.

\section{An Ideal Gas in a Trap}

Let us consider an ideal gas contained by some potential well acting as a trap. This can take place by wall of a container, such that the wall represents positions of a classical turning points. Also the trap can be represented by surrounding particle in a very high densities and pressure case. Quantum mechanically, the particle has a small probability to tunnel past the turning point. So the linear size of the potential trap $L$ has uncertainty $\Delta L$ due to quantum fluctuations, such that it results uncertainty of order $\Delta V=A \Delta L$. We can define the pressure of the gas by the relation

$$
P=2 p \rho v,
$$

where $\rho$ is the gas density and $v$ is the gas particle velocity, and $\rho v$ is the number of collisions per unit time per unit area. According to GUP effect, if we consider $\langle p\rangle \approx p$, the uncertainty in first order approximation will be

$$
\Delta p \Delta L \geq \frac{\hbar}{2}(1-2 \alpha p)
$$

Use (27) to translate the uncertainty in pressure and length to take the form

$$
\Delta P \Delta L \geq \hbar \rho v-\alpha \hbar P .
$$

The mean energy of the gas particle is of order $\sim K T$, so the velocity of the particle can be given as $v=\sqrt{K T / m}$. Equation (29) will take the form

$$
\frac{\Delta P}{P} \Delta L \geq \frac{\hbar}{\sqrt{m K T}}-\alpha \hbar .
$$

Define the thermal wavelength of the particle as $\lambda=$ $\hbar \sqrt{2 \pi / m K T}$ and the uncertainty will take the form

$$
\frac{\Delta P}{P} \frac{\Delta L}{L} \geq \frac{1}{L}\left(\frac{\lambda}{\sqrt{2 \pi}}-\alpha \hbar\right) .
$$


Since the volume $V \sim L^{3}$, then

$$
\frac{\Delta P}{P} \frac{\Delta V}{V} \geq \frac{3}{L}\left(\frac{\lambda}{\sqrt{2 \pi}}-\alpha \hbar\right)
$$

This equation represents the generalized thermodynamic uncertainty principle for an ideal gas in potential trap. It ensures that when the potential trap size is comparable with the thermal wave length, the pressure and the volume cannot be treated as commuting observable. They are playing as quantum mechanical operators acting in wave functions in quantum mechanical approach. This means that we cannot build up the equation of state by a simple relation among $P$, $V$, and $T$, but we must have a wave equation consistence with the new thermodynamical GUP. Since $\alpha \hbar=\alpha_{0} l_{P l}$, (32) will be satisfied at which

$$
\lambda \geq \sqrt{2 \pi} \alpha_{0} l_{P l}
$$

This inequality shows that the thermal wavelength of the particles should be greater than or equal to the minimum length. This naturally assumes a minimum measurable value of thermal wavelength of particles. This result agrees with the discrete nature of the space that has been derived based on the GUP in $[13,14]$. Besides, this result motivates us to suggest that the thermal energy of the particle should not exceed a maximum energy or

$$
K T \leq \frac{1}{m}\left(\frac{M_{P l} c}{\alpha_{0}}\right)^{2}
$$

Furthermore, these derived inequalities prove that the dynamics of the gas particle is not free but restricted by some conditions derived from GUP approach. The appearance of a minimal measurable length and maximum measurable momentum or energy, in (33) and (34), is expected in this quantum gravity approach as a restriction on observable measurement. Finally, (32) shows that when the trap size is much greater that the thermal wavelength, the thermodynamic uncertainty breaks down and drives us to go to the usual observables.

\section{Gas Photon}

In this section, we aim to determine the thermal properties of the radiation field in the presence of GUP. With quantum gravity effects, the photon obeys the dispersion relation:

$$
E=c p(1-\alpha p) \text {. }
$$

Since photons are gauge bosons with a spin $s=1$ and only two possible orientations, the degeneracy factor is therefore $g=2$. Also the number of photons is not conserved and there is no condition on the number of photons, so the canonical partition function, according to Bose-Einstein statistics, is given by

$$
\begin{aligned}
\ln z & =\frac{-V}{\pi^{2} \hbar^{2}} \int \ln \left(1-e^{-\beta E}\right) \frac{p^{2} d p}{(1-\alpha p)^{4}} \\
& =\frac{V \beta c^{2}}{3 \pi^{2} \hbar^{3}} \int \frac{d E}{e^{\beta E}-1}\left(E^{3}+\frac{6 \alpha}{c} E^{4}\right) \\
& =V\left[\frac{\pi^{2} K^{3}}{45 \hbar^{3} c^{3}} T^{3}+48 \alpha \frac{\xi(5) K^{5}}{\pi^{2} \hbar^{3} c^{4}} T^{4}\right],
\end{aligned}
$$

where $\xi$ is the zeta function, and the free energy is

$$
F=-K T \ln z=-V\left[\frac{4 \sigma}{3 c} T^{4}+48 \alpha \frac{\xi(5) K^{5}}{\pi^{2} \hbar^{3} c^{4}} T^{5}\right],
$$

where $\sigma=\left(\pi^{2} K^{4}\right) /\left(60 \hbar^{3} c^{2}\right)$ is the Stefan-Boltzmann constant. The free energy can be rewritten in a standard form as

$$
F=-V \frac{4}{3 c} \sigma_{\mathrm{eff}}(\alpha, T) T^{4},
$$

where the thermal dependance Stefan-Boltzmann constant is

$$
\sigma_{\mathrm{eff}}(\alpha, T)=\sigma\left(1+1260 \alpha \frac{\xi(5) K}{\pi^{4} c} T\right)
$$

Using the partition functions, we determine all thermodynamical properties of the photon gas. Entropy and internal energy density, respectively, are as follows:

$$
\begin{array}{r}
S=-\left.\frac{\partial F}{\partial T}\right|_{T}=V\left[\frac{16 \sigma}{3 c} T^{3}+240 \alpha \frac{\xi(5) K^{5}}{\pi^{2} \hbar^{3} c^{4}} T^{4}\right] \\
u=\frac{F+T S}{V}=\frac{4 \sigma}{c} T^{4}+192 \alpha \frac{\xi(5) K^{5}}{\pi^{2} \hbar^{3} c^{4}} T^{5} .
\end{array}
$$

It is clear from the above two expressions that the entropy and internal energy grows faster than the case of special relativity results with the temperature. This modification is expected where the total number of available microstates of the system is a direct measurement of entropy as well as the internal energy. The pressure of the radiation is

$$
P=-\left.\frac{\partial F}{\partial V}\right|_{T}=\frac{4 \sigma}{3 c} T^{4}+48 \alpha \frac{\xi(5) K^{5}}{\pi^{2} \hbar^{3} c^{4}} T^{5} .
$$

This is an interesting result which is not like the case of ideal gases in which the pressure does not change at the Planck scale. But introducing a new statistical distribution (Bose-Einstein statistic) leads to an appearance of quantum gravity effect as an increase in pressure. Indeed, the increase in repulsive force among the particle gas entails the increase in pressure. The specific heat is a suitable measurable quantity that can be detected experimentally. The specific heat of photon gas is

$$
C_{V}=\left.T \frac{\partial S}{\partial T}\right|_{V}=V\left[\frac{16 \sigma}{c} T^{3}+960 \alpha \frac{\xi(5) K^{5}}{\pi^{2} \hbar^{3} c^{4}} T^{4}\right] .
$$


As a consequence of an elevation in entropy and internal energy, specific heat is increased in comparison with special relativistic one. In order to obtain pressure-energy density relation divide (42) in (41); one gets

$$
\frac{P}{u}=\frac{1}{3}-240 \alpha \frac{\xi(5) K}{\pi^{4} c} T .
$$

The usual relation $u=3 p$ is recovered after taking $\alpha=0$. To obtain an equation of state one has to write the temperature as a power series of $\alpha$. Solving (41), keeping only first order of approximation in $\alpha$, one gets

$$
T=\left(\frac{c}{4 \sigma}\right)^{1 / 4} u^{1 / 4}-360 \alpha \frac{\xi(5) K}{\pi^{4}}\left(\frac{1}{c \sigma}\right)^{1 / 2} u^{1 / 2} .
$$

Substitute (45) into (44) one can obtain the first order approximation in equation of state

$$
P=\frac{u}{3}\left[1-720 \alpha \frac{\xi(5)}{\pi^{4} c}\left(\frac{15 \hbar^{3} c^{3}}{\pi^{2}}\right)^{1 / 4} u^{1 / 2}\right]
$$

Equation (46) represents a modified equation of state due to the generalized uncertainty principle.

\section{Conclusions}

Various approaches to quantum gravity such as string theory, black hole physics, and doubly special relativity predict a considerable modification in Heisenberg uncertainty principle to be a generalized uncertainty principle. This modification leads to a change in the energy-momentum dispersion relation and in the physical phase space. These lead to a considerable enhancement in the number of accessible microscopic states of the phase space volume with GUP effects. In this paper we investigate the effect of the GUP in thermodynamic properties of ideal and photon gases. An analytical expression of the partition function for massive ideal gas and photon gas is derived. Using the modified partition function, we determined the thermodynamic functions such as free energy, entropy, pressure, internal energy, and specific heat. We found that there is a considerable increase in these quantities in comparison with the corresponding relativistic quantities. This is due to an increase in accessible microscopic states in the phase space which leads to an increase in entropy that carries a physical properties of the system. This in turn leads to an increase in thermodynamic properties of the system. The pressure of the ideal gas does not change, but the pressure of the photon gas is increased. Another problem is considered and we study the effect of GUP in a gas contained in a potential trap. When the size of the potential trap is comparable with the thermal wavelength of the particles, the pressure and the volume cannot be treated as commuting observable. Quantum gravity puts some restrictions on the particle dynamics, where we found that the thermal wavelength of the particles should be greater than, or equal to, a minimum length and this definitely agrees with the discrete nature of the space that has been derived based on GUP in previous studies. Besides, it is found that the thermal energy of the particle of the ideal gas should not exceed a maximum energy. The results we obtained in this paper could be useful to study the effect of the GUP with astrophysical objects such as standard model of stars (photon plus nonrelativistic ideal gas) [51], white dwarfs (degenerate electron gas) [51], and neutron stars (Oppenheimer-Volkoff model: degenerate neutron gas) $[52,53]$. We hope to report on these in the future.

\section{Conflict of Interests}

The authors declare that there is no conflict of interests regarding the publication of this paper.

\section{Acknowledgments}

The research of Ahmed Farag Ali is supported by Benha University (http://www.bu.edu.eg/) and CFP in Zewail City, Egypt. The authors would like to thank the anonymous referees for enlightening comments and helpful suggestions which helped improve the quality of the paper.

\section{References}

[1] D. Amati, M. Ciafaloni, and G. Veneziano, "Can spacetime be probed below the string size?” Physics Letters B, vol. 216, no. 1-2, pp. 41-47, 1989.

[2] M. Maggiore, "A generalized uncertainty principle in quantum gravity”, Physics Letters B, vol. 304, no. 1-2, pp. 65-69, 1993.

[3] M. Maggiore, "Quantum groups, gravity, and the generalized uncertainty principle," Physical Review D, vol. 49, no. 10, pp. 5182-5187, 1994.

[4] M. Maggiore, "The algebraic structure of the generalized uncertainty principle," Physics Letters B, vol. 319, no. 1-, pp. 83-86, 1993.

[5] L. J. Garay, "Quantum gravity and minimum length," International Journal of Modern Physics A, vol. 10, p. 145, 1995.

[6] S. Hossenfelder, M. Bleicher, S. Hofmann, J. Ruppert, S. Scherer, and H. Stöcker, "Signatures in the Planck regime," Physics Letters $B$, vol. 575, no. 1-2, pp. 85-99, 2003.

[7] C. Bambi and F. R. Urban, "Natural extension of the generalized uncertainty principle," Classical and Quantum Gravity, vol. 25, no. 9, Article ID 095006, 2008.

[8] F. Scardigli, "Generalized uncertainty principle in quantum gravity from micro-black hole gedanken experiment," Physics Letters B, vol. 452, no. 1-2, pp. 39-44, 1999.

[9] P. F. Mende, "String theory at short distance and the principle of equivalence," Erice Theoretical Physics, pp. 286-299, 1992.

[10] A. Kempf, G. Mangano, and R. B. Mann, "Hilbert space representation of the minimal length uncertainty relation," Physical Review D, vol. 52, no. 2, pp. 1108-1118, 1995.

[11] A. Kempf, "Non-pointlike particles in harmonic oscillators," Journal of Physics A, vol. 30, no. 6, pp. 2093-2101, 1997.

[12] F. Brau, "Minimal length uncertainty relation and the hydrogen atom," Journal of Physics A, vol. 32, no. 44, pp. 7691-7696, 1999.

[13] A. F. Ali, S. Das, and E. C. Vagenas, "Discreteness of space from the generalized uncertainty principle," Physics Letters B, vol. 678, no. 5, pp. 497-499, 2009. 
[14] S. Das, E. C. Vagenas, and A. F. Ali, "Discreteness of space from GUP II: relativistic wave equations," Physics Letters B, vol. 690, no. 4, pp. 407-412, 2010.

[15] J. L. Cortes and J. Gamboa, "Quantum uncertainty in doubly special relativity," Physical Review D, vol. 71, Article ID 065015, 4 pages, 2005.

[16] A. F. Ali, S. Das, and E. C. Vagenas, "Proposal for testing quantum gravity in the lab," Physical Review D, vol. 84 , no. 4 , Article ID 044013, 10 pages, 2011.

[17] A. Tawfik, H. Magdy, and A. F. Ali, "Lorentz invariance violation and generalized uncertainty principle," http://arxiv.org/ abs/1205.5998/.

[18] B. R. Majhi and E. C. Vagenas, "Modified dispersion relation, photon's velocity, and Unruh effect," Physics Letters B, vol. 725, no. 4-6, pp. 477-480, 2013.

[19] S. Das and E. C. Vagenas, "Universality of quantum gravity corrections," Physical Review Letters, vol. 101, no. 22, Article ID 221301, 2008.

[20] S. Das and E. C. Vagenas, "Phenomenological implications of the generalized uncertainty principle," Canadian Journal of Physics, vol. 87, no. 3, pp. 233-240, 2009.

[21] W. Chemissany, S. Das, A. F. Ali, and E. C. Vagenas, "Effect of the generalized uncertainty principle on post-inflation preheating," Journal of Cosmology and Astroparticle Physics, vol. 12, Article ID 017, 2011.

[22] M. Sprenger, M. Bleicher, and P. Nicolini, "Neutrino oscillations as a novel probe for a minimal length," Classical and Quantum Gravity, vol. 28, no. 23, Article ID 235019, 2011.

[23] A. F. Ali, "No existence of black holes at LHC due to minimal length in quantum gravity," Journal of High Energy Physics, vol. 2012, article 67, 2012.

[24] A. F. Ali, H. Nafie, and M. Shalaby, "Minimal length, maximal energy and black-hole remnant," Europhysics Letters, vol. 100, no. 2, article 20004, 2012.

[25] A. F. Ali, "Minimal length in quantum gravity, equivalence principle and holographic entropy bound," Classical and Quantum Gravity, vol. 28, no. 6, Article ID 065013, 2011.

[26] B. Majumder and S. Sen, "Do the modified uncertainty principle and polymer quantization predict same physics?" Physics Letters $B$, vol. 717, no. 4-5, pp. 291-294, 2012.

[27] K. Nozari and S. Saghafi, " Natural cutoffs and quantum tunneling from black hole horizon," Journal of High Energy Physics, vol. 2012, article 5, 2012.

[28] B. Majumder, "Effects of the modified uncertainty principle on the inflation parameters," Physics Letters B, vol. 709, no. 3, pp. 133-136, 2012.

[29] K. Nozari and A. Etemadi, "Minimal length, maximal momentum, and Hilbert space representation of quantum mechanics," Physical Review D, vol. 85, no. 10, Article ID 104029, 12 pages, 2012.

[30] I. Pikovski, M. R. Vanner, M. Aspelmeyer, M. S. Kim, and Č. Brukner, "Probing planck-scale physics with quantum optics," Nature Physics, vol. 8, no. 5, pp. 393-397, 2012.

[31] F. Marin, F. Marino, M. Bonaldi et al., "Gravitational bar detectors set limits to Planck-scale physics on macroscopic variables," Nature Physics, vol. 9, pp. 71-73, 2013.

[32] J. D. Bekenstein, "Is a tabletop search for Planck scale signals feasible?” Physical Review D, vol. 86, no. 12, Article ID 124040, 9 pages, 2012.

[33] J. D. Bekenstein, "Can quantum gravity be exposed in the laboratory?: a tabletop experiment to reveal the quantum foam," in Proceedings of the Keynote Lecture at the International Workshop on Horizon of Quantum Physics: From Foundations to Quantum Enabled Technologies, Taipei, Taiwan, October 2012.

[34] A. Camacho and A. Macías, "Thermodynamics of a photon gas and deformed dispersion relations," General Relativity and Gravitation, vol. 39, no. 8, pp. 1175-1183, 2007.

[35] O. Bertolami and C. A. D. Zarro, "Towards a noncommutative astrophysics," Physical Review Ds, vol. 81, no. 2, Article ID 025005, 2010.

[36] G. Amelino-Camelia, "Proposal of a second generation of quantum-gravity-motivated lorentz-symmetry tests: Sensitivity to effects suppressed quadratically by the planck scale," International Journal of Modern Physics D, vol. 12, no. 9, pp. 1633-1639, 2003.

[37] M. Gregg and S. A. Major, "On modified dispersion relations and the chandrasekhar mass limit," International Journal of Modern Physics D, vol. 18, no. 6, pp. 971-982, 2009.

[38] S. Das, S. Ghosh, and D. Roychowdhury, "Relativistic thermodynamics with an invariant energy scale," Physical Review D, vol. 80, no. 12, Article ID 125036, 6 pages, 2009.

[39] G. Amelino-Camelia, "Special treatment," Nature, vol. 418, no. 6893, pp. 34-35, 2002.

[40] G. Amelino-Camelia, "Testable scenario for relativity with minimum length," Physics Letters B, vol. 510, no. 1-4, pp. 255263, 2001.

[41] G. Amelino-Camelia, "Relativity in spacetimes with shortdistance structure governed by an observer-independent (Planckian) length scale," International Journal of Modern Physics D, vol. 11, no. 1, pp. 35-59, 2002.

[42] J. Magueijo and L. Smolin, "Lorentz invariance with an invariant energy scale," Physical Review Letters, vol. 88, no. 19, pp. 1904031-1904034, 2002.

[43] J. Magueijo and L. Smolin, "String theories with deformed energy-momentum relations, and a possible nontachyonic bosonic string," Physical Review D, vol. 71, no. 2, Article ID 026010, 6 pages, 2005.

[44] S. Das and D. Roychowdhury, "Thermodynamics of a photon gas with an invariant energy scale," Physical Review D, vol. 81, no. 8, Article ID 085039, 7 pages, 2010.

[45] N. Chandra and S. Chatterjee, "Thermodynamics of ideal gas in doubly special relativity," Physical Review D, vol. 85, no. 4, Article ID 045012, 9 pages, 2012.

[46] K. Nozari and S. H. Mehdipour, "Implications of minimal length scale on the statistical mechanics of ideal gas," Chaos, Solitons and Fractals, vol. 32, no. 5, pp. 1637-1644, 2007.

[47] K. Nozari and B. Fazlpour, "Generalized uncertainty principle, modified dispersion relations and the early universe thermodynamics," General Relativity and Gravitation, vol. 38, no. 11, pp. 1661-1679, 2006.

[48] R. K. Pathria, Statistical Mechanics, Butterworth-Heinemann, Oxford, UK, 1996.

[49] W. Greiner, L. Neise, and H. Stoecker, Thermodynamics and Statistical Mechanics.

[50] I. S. Gradshteyn and I. M. Ryzhik, Table of Integrals, Series, and Products, Elsevier Academic Press, 7th edition, 2007.

[51] S. Chandrasekhar, An Introduction to the Study of Stellar Structure, Dover, Mineola, NY, USA, 1967.

[52] J. R. Oppenheimer and G. M. Volkoff, "On massive neutron cores," Physical Review, vol. 55, no. 4, pp. 374-381, 1939.

[53] O. Bertolami and C. A. D. Zarro, "Towards a noncommutative astrophysics," Physical Review D, vol. 81, no. 2, Article ID 025005, 2010. 

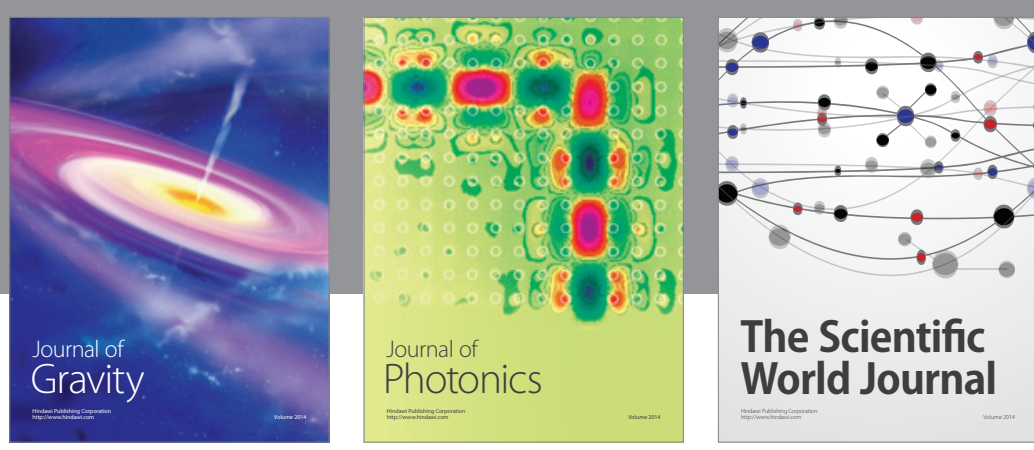

The Scientific World Journal
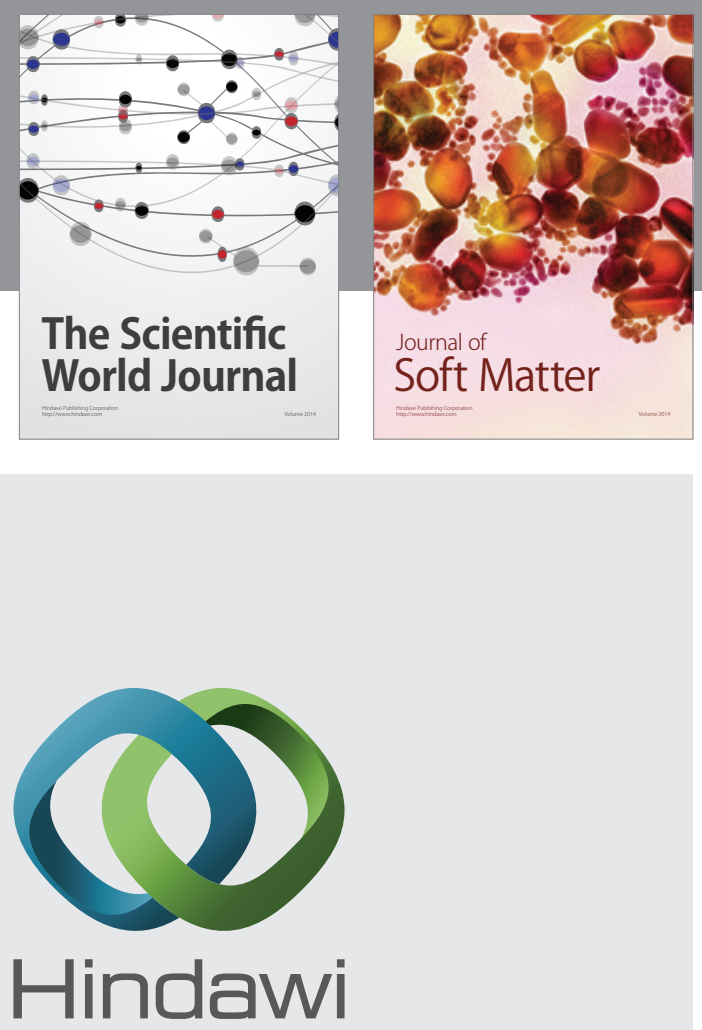

Submit your manuscripts at

http://www.hindawi.com

nternational Journal of

Statistical Mechanics
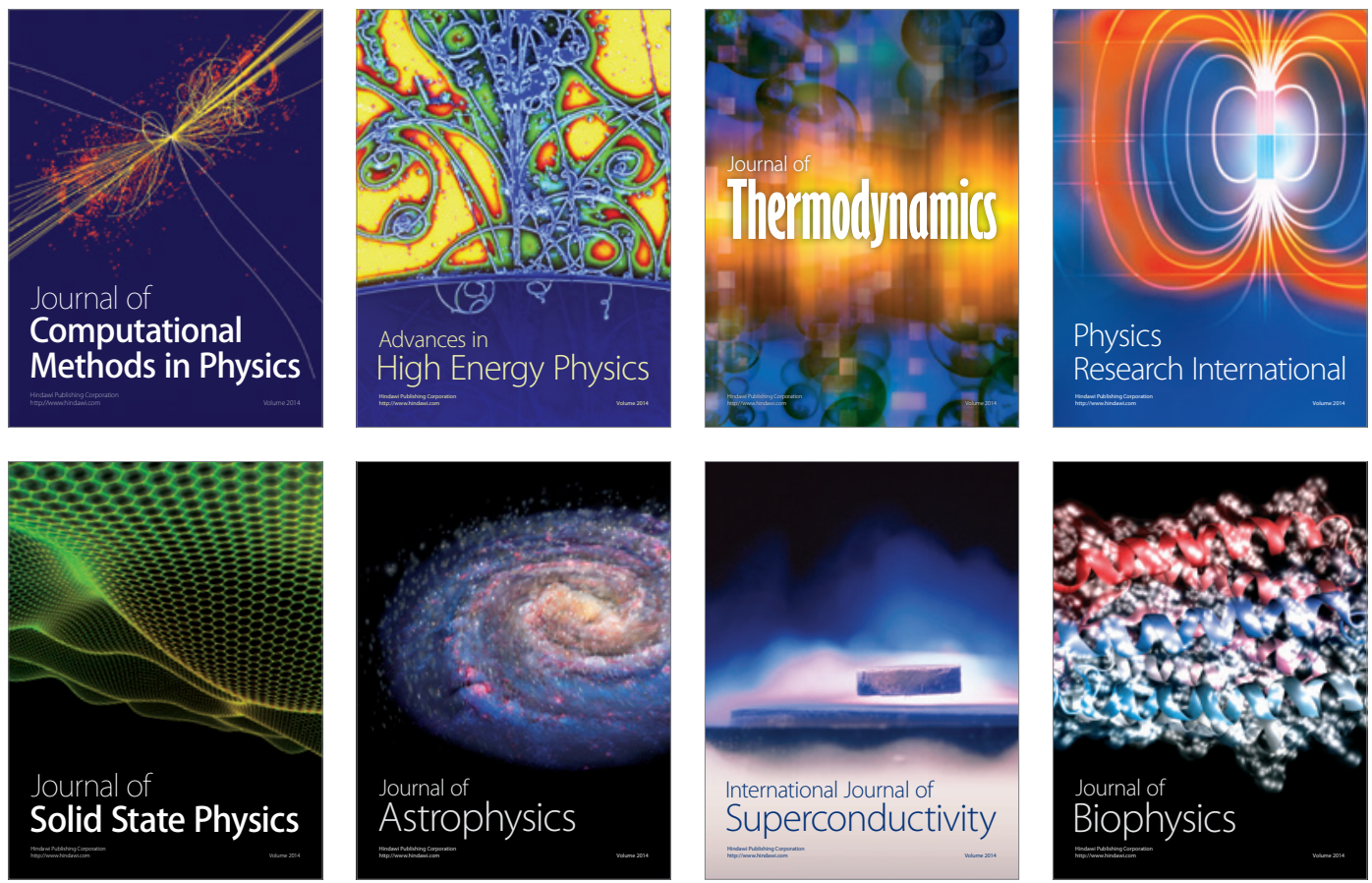
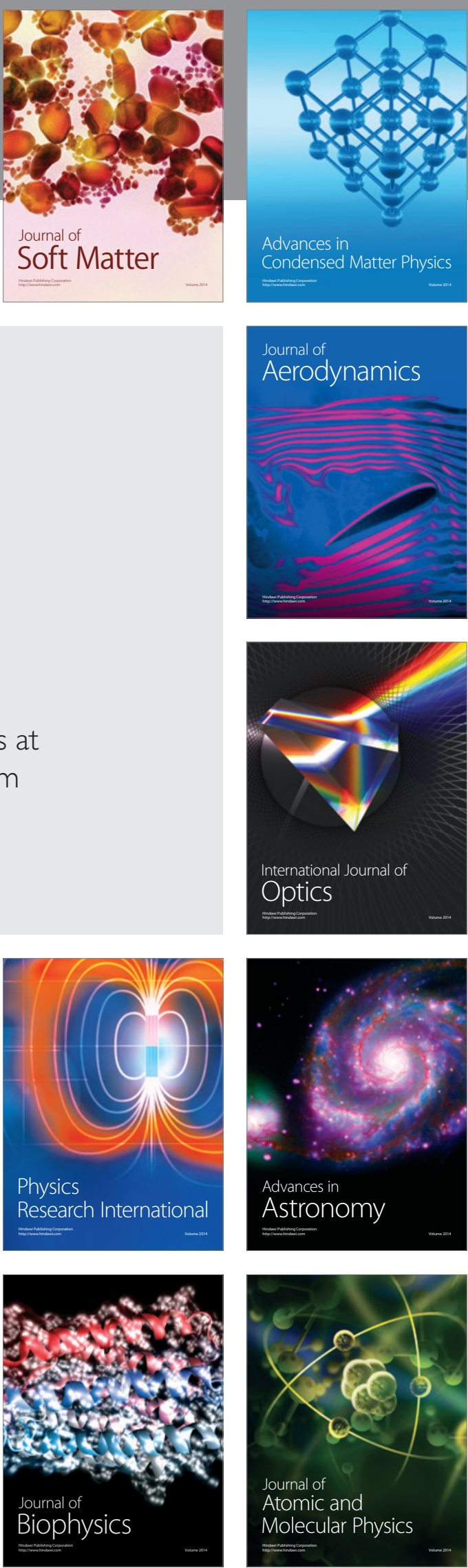